\title{
Stabilization Method of Islanded DC Microgrid Based on the Exact Feedback Linearization
}

\author{
Zhonglin Yang ${ }^{1,2, a}$, Xiaoming Zha ${ }^{1, b}$ \\ ${ }^{1}$ School of Electrical Engineering, Wuhan University, Wuhan, 430072, China \\ ${ }^{2}$ School of Electrical Engineering, Naval University of Engineering, Wuhan, 430033, China \\ aemail: blueduny@sina.com, bemail: xmzha@whu.edu.cn
}

Keywords: DC microgrid ; Stabilization; Nonlinear state feedback;Exact feedback linearzation

\begin{abstract}
There are a lot of converters in the DC microgrid. These tightly regulated closed-loop converters may cause stability problem of bus voltage when used as a load. In order to improve the stability of the DC microgrid, a novel stabilization method based on exact feedback linearization is proposed. The nonlinear system is transformed into a linear system, and the large signal stability control is realized by using the exact linearization technology. The feasibility and effectiveness of this method are verified by the simulation results.
\end{abstract}

\section{Introduction}

In recent years, under the dual pressure of energy demand and environmental protection, the micro grid has gained more and more attention and application [1][2]. Compared to AC microgrid, DC micro grid which has the advantages of high efficiency, simple control, high reliability and good power quality, etc., is gradually being increasingly concerned about [3][4]. However, a large number of power electronic devices in the DC microgrid, which is characterized by constant power load, may cause the instability of the DC bus voltage of the DC micro grid [5][6].

A method for improving the stability of DC microgrid by introducing the virtual capacitor into the load point is proposed[7]. The papers [8] proposed the method to improve the stability of the DC microgrid by modify the circuit structure or parameters of DC microgrid. In the paper [9], the equivalent impedance of the converter which was connected to the Grid was changed by increasing the active damping signal, which can improve the stability of the DC microgrid. A method for improving the voltage stability of the DC micro grid is proposed by improving the PID control [10]. All these methods above all were the small signal stability at the equilibrium point. In this paper, the large signal stability of DC microgrid is realized by the introduction of the exact feedback linearization technique.

\section{System model and its stability analysis}

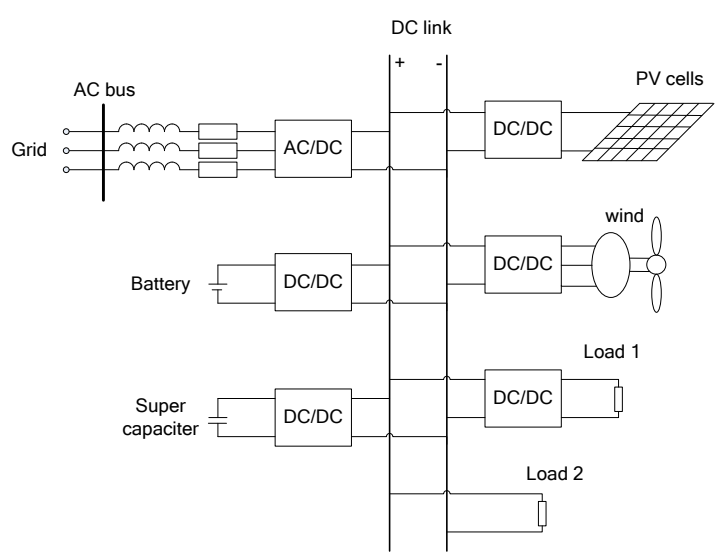

Fig. 1 Schematic diagram of a dc microgrid

Typical structure of the DC microgrid is shown in Fig.1, which contains a large number of power electronic converters. When the grid faults occur, the rectifier connected to the grid stops working, 
the DC bus voltage can be maintained by the DC/DC converter connected to the energy storage unit. When the load point converter works in the constant voltage mode and the control performance is good, the load point converter and its load relative to the DC microgrid as the constant power load (CPL) [11]. Under normal weather conditions, the distributed power, such as the photovoltaic and wind power, is generally working in the maximum power tracking (MPPT) mode, at this time of the photovoltaic and wind power and its converter can be seen as a constant power [12].

To analyze simply, the case in which the Boost converter was used to maintain the DC microgrid bus voltage was taken as the example to study system stability. The simplified model of the DC microgrid is shown in Fig.2.

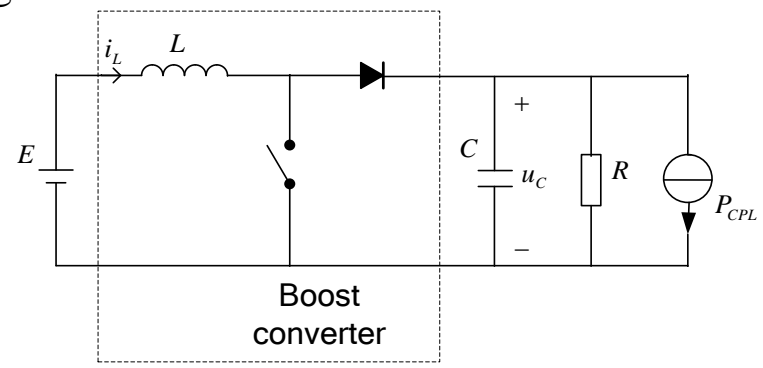

Fig. 2 The simpled model of a dc microgrid in islanded model

Where $E$ is the input voltage of Boost converter, $L$ is the filter inductor of the Boost converter, $C$ is the equivalent capacitance of all the capacitances which connect to the DC bus, $R$ is equivalent resistance of all the resistant load, and the $P_{\mathrm{CPL}}$ is the equivalent constant power load, which value is the sum of the input power of all the constant power loads subtract the sum of all the output power of the constant power sources.

By using the state average method, the circuit equation can be written as

$$
\left\{\begin{array}{l}
L \frac{d i_{L}}{d t}=E-(1-d) u_{C} \\
C \frac{d u_{C}}{d t}=(1-d) i_{L}-\frac{u_{C}}{R}-\frac{P_{C P L}}{u_{C}}
\end{array}\right.
$$

Where $d$ is the duty cycle of the Boost converter. By linearization to the equation (1) at its equilibrium point, the stability condition can be obtain [13]

$$
P_{C P L}<\frac{U_{C}^{2}}{R}
$$

From the equation (2), it can be seen that in order to make the DC microgrid stable, the power of the constant power load in the system must be less than that of the resistive load. However, a typical DC microgrid contains about $80 \%-85 \%$ of the constant power load, the $15 \%-20 \%$ of the resistive load [10]. So in order to improve the stability of DC microgrid, some measures must be taken.

\section{State feedback exact linearization of single input single output system}

A single input single output affine nonlinear system can be expressed as follows in the standard form [15]

$$
\left\{\begin{array}{l}
\dot{X}=f(X)+g(X) u \\
y=h(X)
\end{array}\right.
$$

Where $X$ is the state variable vector of the system, $u$ is the input of the system and $y$ is the output of the system.

When the system's relationship degree is equal to the order number of the system, it can be transfer to the linear system (5) by the following coordinate transformation

$$
Z=\left[\begin{array}{c}
z_{1} \\
z_{2} \\
\vdots \\
z_{n}
\end{array}\right]=\left[\begin{array}{c}
h(X) \\
L_{f} h(X) \\
\vdots \\
L_{f}^{n-1} h(X)
\end{array}\right]
$$




$$
\dot{Z}=A Z+B v
$$

Where $Z$ is the state vector for the transformed system, $v$ is the control variable, $L_{f} h(X)$ is the $\mathrm{Li}$ derivatives of $h(X)$ relative to $f$, and

$$
A=\left[\begin{array}{cccccc}
0 & 1 & 0 & \cdots & 0 & 0 \\
0 & 0 & 1 & \cdots & 0 & 0 \\
\vdots & \vdots & \vdots & \ddots & \vdots & \vdots \\
0 & 0 & 0 & \cdots & 1 & 0 \\
0 & 0 & 0 & 0 & 0 & 0
\end{array}\right], \quad B=\left[\begin{array}{c}
0 \\
0 \\
\vdots \\
0 \\
1
\end{array}\right]
$$

The relationship between the input control variable $u$ of the original nonlinear system and the control variable of the transformed linear system (5) is

$$
u=-\frac{L_{f}{ }^{n} h(X)}{L_{g} L_{f}{ }^{n-1} h(X)}+\frac{1}{L_{g} L_{f}{ }^{n-1} h(X)} v
$$

By using the linear optimal control theory, the optimal control variable of linear system (5) can be known

$$
v=-R^{-1} B^{T} P^{*} Z(t)
$$

Where $R$ is weight coefficient matrix and equal to 1. $P^{*}$ is solution of the following Riccati matrix equation

$$
A^{T} P+P A-P B R^{-1} B^{T} P+Q=0
$$

The linear optimal control theory [14] points out: the optimal control system is a large signal stability system.

\section{Stabilization method of DC microgrid based on exact feedback linearization}

Supposed $x_{1}=i_{\mathrm{L}}, x_{2}=u_{\mathrm{C}}$, the system (1) is adapted to the form of the following affine nonlinear systems

$$
\left[\begin{array}{c}
\dot{x}_{1} \\
\dot{x}_{2}
\end{array}\right]=\left[\begin{array}{c}
\frac{E}{L}-\frac{x_{2}}{L} \\
\frac{x_{1}}{C}-\frac{x_{2}}{R C}-\frac{P_{C P L}}{C x_{2}}
\end{array}\right]+\left[\begin{array}{c}
\frac{x_{2}}{L} \\
\frac{x_{1}}{C}
\end{array}\right] d
$$

so

$$
f(X)=\left[\begin{array}{c}
\frac{E}{L}-\frac{x_{2}}{L} \\
\frac{x_{1}}{C}-\frac{X_{2}}{R C}-\frac{P_{C P L}}{C x_{2}}
\end{array}\right] \quad g(X)=\left[\begin{array}{c}
\frac{x_{2}}{L} \\
\frac{x_{1}}{C}
\end{array}\right]
$$

Supposed

$$
h(X)=\frac{x_{1}^{2}-I_{r e f}^{2}}{C}+\frac{x_{2}^{2}-U_{r e f}^{2}}{L}
$$

Where $U_{\text {ref }}$ is the reference value of DC bus voltage, $I_{\text {ref }}$ is the stable value of inductor current which can be calculate by following equation

$I_{r e f}=\frac{U_{r e f}^{2}}{R E}+\frac{P_{C P L}}{E}$

Because of

$$
\begin{gathered}
L_{g} h(X)=\frac{2 x_{1} x_{2}}{L C}-\frac{2 x_{1} X_{2}}{L C}=0 \\
L_{g} L_{f} h(X)=\frac{2 E x_{2}}{L^{2} C}+\frac{4 x_{1} x_{2}}{L C^{2} R} \neq 0
\end{gathered}
$$

It can be seen that the system's relationship degree is two which equal to the order number of the system. So, the nonlinear system can realize the exact linearization by nonlinear state feedback. 


\section{Supposed}

$$
\left\{\begin{array}{l}
z_{1}=h(X)=\frac{x_{1}{ }^{2}-I_{r e f}{ }^{2}}{C}+\frac{x_{2}{ }^{2}-U_{r e f}{ }^{2}}{L} \\
z_{2}=L_{f}(X)=\frac{2 E x_{1}}{L C}-\frac{2 x_{2}{ }^{2}}{L C R}-\frac{2 P_{C P L}}{L C}
\end{array}\right.
$$

It can be obtain that

$$
\begin{aligned}
& {\left[\begin{array}{l}
\dot{z}_{1} \\
\dot{z}_{2}
\end{array}\right]=\left[\begin{array}{ll}
0 & 1 \\
0 & 0
\end{array}\right]\left[\begin{array}{l}
z_{1} \\
z_{2}
\end{array}\right]+\left[\begin{array}{l}
0 \\
1
\end{array}\right] v} \\
& v=L_{f}{ }^{2} h(X)+L_{g} L_{f} h(X) \cdot d
\end{aligned}
$$

where

$$
L_{f}{ }^{2} h(X)=\frac{2 E^{2}-2 E x_{2}}{L^{2} C}+\frac{2 P_{C P L}-2 x_{1} x_{2}}{L C^{2} R}+\frac{2 x_{2}^{2}}{L C^{2} R^{2}}
$$

For linear systems (16), the optimal control method of the quadratic performance index is adopted to obtain the control variables of the system.

The performance index can be selected in the following form

$$
J=\int_{0}^{\infty}\left[Z^{T}(t) Q Z^{T}(t)+v^{2}(t)\right] d t
$$

According to the optimal control theory, the optimal control solution $v$ for the above performance index is the linear feedback of the state variables

$$
v=-K Z(t)=-k_{1} z_{1}-k_{2} z_{2}
$$

The feedback gain matrix can be obtained by solving the Riccati equation according to the selected weight matrix $Q$.

From equation (17) and equation (19), the duty cycle of Boost converter can be obtained in following form

$$
d=-\frac{L_{f}{ }^{2} h(X)+k_{1} z_{1}+k_{2} z_{2}}{L_{g} L_{f} h(X)}
$$

Where $z_{1}$ and $z_{2}$ can be obtained from equation (15).

As can be seen from the above, the final control law is a complex nonlinear function of the state vector.

\section{The Simulation experiment}

In order to verify the effectiveness of the proposed method, the paper uses Matlab/Simulink to build a simulation model of the DC microgrid, and its system structure is shown in Fig.3.

DC bus

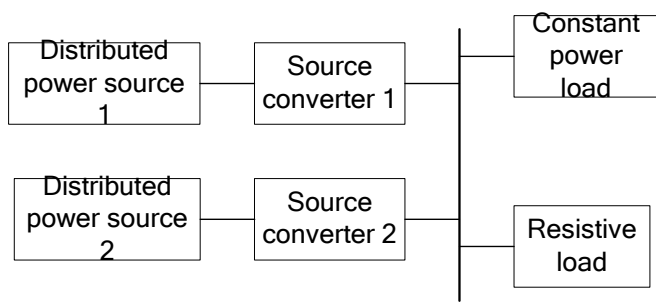

Fig. 3 The DC microgrid with two generators and two loads

The source converter 1 was the Boost converter which maintains the DC bus voltage. The source converter 2 was the converter for constant power control.

The parameters of simulation system are as follows: $E=150 \mathrm{~V}, L=8 \mathrm{mH}, C=0.5 \mathrm{mF}$, and $U_{\text {ref }}=200 \mathrm{~V}$. The output power of the constant power source was $500 \mathrm{~W}$, and the power of CPL was $2500 \mathrm{~W}$.So the value of the equivalent constant power load $P_{\mathrm{CPL}}$ was $2000 \mathrm{~W}$. The value of resistive load R was $50 \Omega$. The switching frequency of the converter was $10 \mathrm{kHz}$. Simulation results are shown in the Fig. 4 and Fig. 5. 
When the system did not join the feedback control loop, the eigenvalues at the equilibrium point (200V,15A) are $25 \pm$ j374.17 by calculation from (1). The bus voltage $u_{\mathrm{C}}$ and the Boost converter inductor current $i_{\mathrm{L}}$ were shown in Fig. 4. Because the real part of the eigenvalues are greater than zero, the bus voltage $u_{\mathrm{C}}$ and inductor current $i_{\mathrm{L}}$ diverge. Until the inductor current decreased to zero, the bus voltage maintains greatly oscillation.

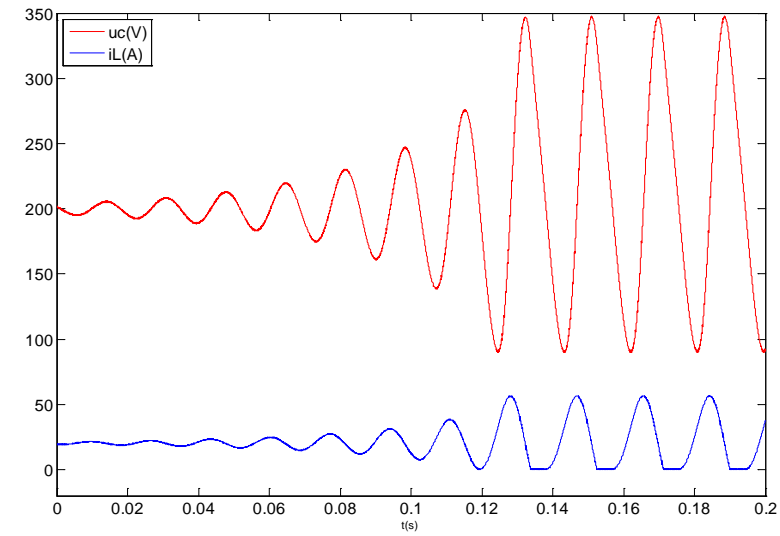

Fig.4 The bus voltage and inductance current of DC microgrid supported by Boost converter without the nonlinear feedback

It was pointed out in the paper [15] that in order to make sure the stability of original nonlinear system, the time constant of the linear system with the optimal control need to be equivalent to that of the original nonlinear system. Because the natural frequency of the Boost converter is $\omega_{0}=\frac{1-D}{\sqrt{L C}}$, so here $Q=10^{11}, \quad R=1$, and it can be obtained that $k_{1}=316228, k_{2}=795$. At this time, the time constant of the linear system equals to that of the original nonlinear system, which guarantee the system stably.

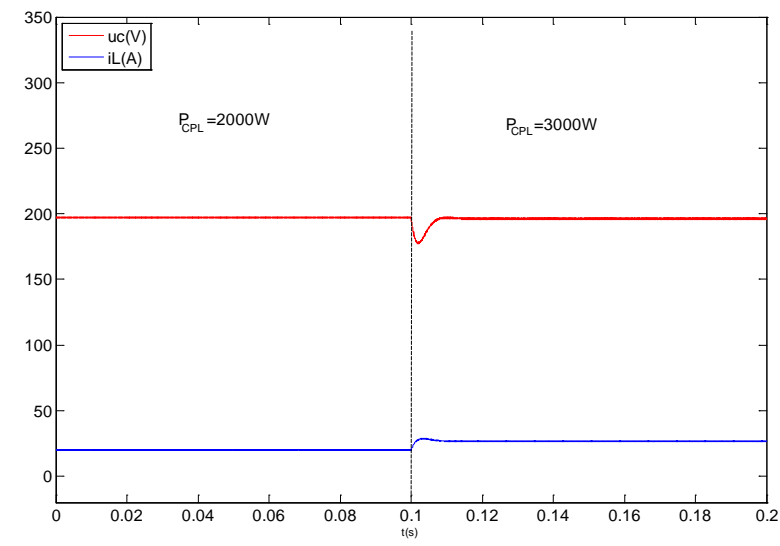

Fig.5 The bus voltage and inductance current of DC microgrid supported by Boost converter with the nonlinear feedback

DC microgrid bus voltage $u_{\mathrm{C}}$ and Boost converter inductor current $i_{\mathrm{L}}$ waveform as shown in Fig.5. At the time of $0.1 \mathrm{~s}$, the total power of constant power load was changed from $2000 \mathrm{~W}$ to $3000 \mathrm{~W}$, and the DC bus voltage can be quickly recovered, which shows that the exact feedback linearization technique can not only guarantee the stability of the system, but also improve the dynamic performance of the system.

From the above experimental results, it can be known that the exact feedback linearization of the DC microgrid can be achieved by using the nonlinear state feedback technique, which can overcome the adverse effect of the constant power load and make sure the stability of the DC microgrid. 


\section{Conclusion}

In this paper, the negative effect of the constant power load is offset by the nonlinear state feedback and optimal control, and the damping of the system is increased by using the exact feedback linearization technique. The simulation results verify the validity of the proposed method.

\section{References}

[1] Xiaonan Lu, Josep M. Guerrero, Kai Sun, et al. An Improved Droop Control Method for DC Microgrids Based on Low Bandwidth Communication with DC Bus Voltage Restoration and Enhanced Current Sharing Accuracy[J], IEEE Transactions on Power Electronics, 2014,29(4):1800-1812.

[2] Xisheng Tang, Wei Deng, Zhiping Qi. Investigation of the Dynamic Stability of Microgrid[J] . IEEE Transactions on Power Systems, 2014,29(2):698-706.

[3] Sandeep Anand, B. G. Fernandes. Reduced-Order Model and Stability Analysis of Low-Voltage DC Microgrid[J]. IEEE Transactions on Industrial Electronics, 2013,60(11):5040-5049.

[4] Xiaonan Lu, Kai Sun, Josep M. Guerrero, et al. State-of-Charge Balance Using Adaptive Droop Control for Distributed Energy Storage Systems in DC Microgrid Applications[J]. IEEE Transactions on Industrial Electronics, 2014,61(6):2804-2815.

[5] Hao Yuchen, Wu Zaijun, Dou Xiaobo, et al. Application of Multi-agent Systems to the DC Microgrid Stability Control[J], Proceeding of the CSEE, 2012, 9(32):27-34.

[6] Xiaogang Feng, Jinjun Liu, Fred C. Lee.Impedance Specifications for Stable DC Distributed Power Systems[J], IEEE Transactions on Power Electronics, 2002, 17(2): 157-162.

[7] Pierre Magne, Didier Marx, Babak Nahid-Mobarakeh. Large-signal stabilization of a DC-link supplying a constant power load using a virtual capacitor: Impact on the Domain of Attraction[J].IEEE Transactions on Industry Applications, 2012,48( 3):878-887.

[8] Amr Ahmed A. Radwan, Yasser Abbel-Rady I. Mohamed. Linear active stabilization of converter-dominated DC microgrids[J], IEEE Transactions on Smart Grid, 2012, 3(1):203-216.

[9] Li Yumei, Zha Xiaoming, Liu Fei, Sun Jianjun. Stability Control Strategy for DC Microgrid with Constant Power Load[J]. Electric Power Automation Equipment, 2014, 34(8): 57-64(in Chinese).

[10] P. Liutanakul, A. Awan, S. Pierfederici, B. Nahid-Mobarakeh, etc. Linear stabilization of a dc bus supplying a constant power load: A general design approach[J], IEEE Transaction on Power Electronics, 2010, 25(2): 475-488.

[11] Andre P. N. Tahim, Daniel J. Pagano, Eduardo Lenz, et al. Modeling and Stability Analysis of Islanded DC Microgrids under Droop Control [J], IEEE Transaction on Power Electronics, 2010, 25(2): 475-488.

[12] A. Emadi, B. Fahimi, M. Ehsani. On the concept of negative impedance instability in advanced aircraft power systems with constant power loads[C], The 34th Intersociety Energy Convers. Eng. Conference, Vancouver, BC, Canada, Aug, 1999:1-11.

[13] Hu Yueming. Theory and applications of nonliear control sustems[M]. Beijing: Nation Defence Industry Publisher, 2005:68-100(in Chinese).

[14] Lu Qiang, Sun Yuanzhang. Nonliear control of power system[M]. Beijing: Science Press, 1993:1-164(in Chinese). 
[15] Exact feedback linearization control to Buck converter with constant power loads[J]. Control and Decision, 2009,7(24):1117-1120(in Chinese). 